
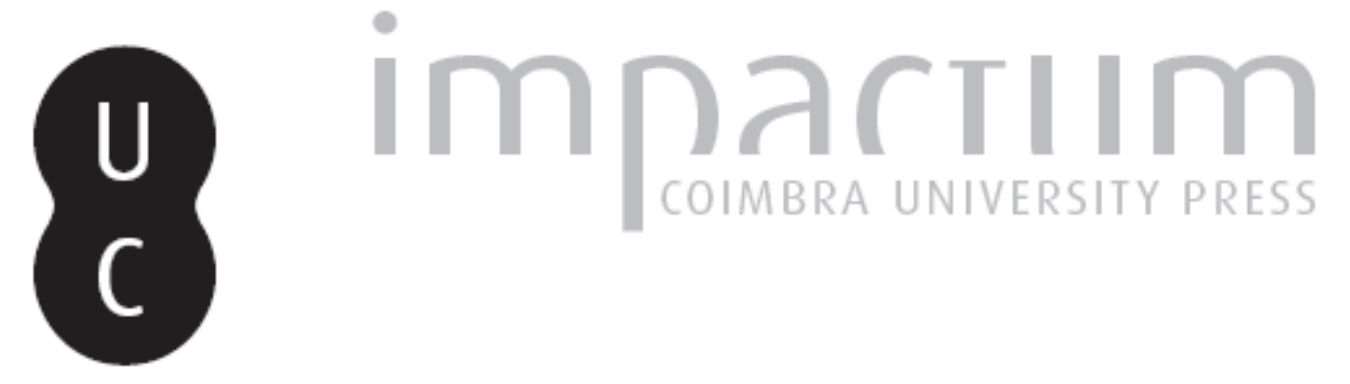
Utilização da análise multi-níveis para avaliação da vulnerabilidade da população da
AML ao calor

Autor(es): $\quad$ Andrade, Henrique; Nogueira, Helena; Canário, Paulo

Publicado por: $\begin{aligned} & \text { Faculdade de Letras da Universidade de Coimbra, Departamento de } \\ & \text { Geografia }\end{aligned}$

URL

persistente:

URI:http://hdl.handle.net/10316.2/30229

DOI: $\quad$ DOI:http://dx.doi.org/10.14195/0871-1623_32_21

Accessed : $\quad$ 26-Apr-2023 15:40:49

A navegação consulta e descarregamento dos títulos inseridos nas Bibliotecas Digitais UC Digitalis, UC Pombalina e UC Impactum, pressupõem a aceitação plena e sem reservas dos Termos e Condições de Uso destas Bibliotecas Digitais, disponíveis em https://digitalis.uc.pt/pt-pt/termos.

Conforme exposto nos referidos Termos e Condições de Uso, o descarregamento de títulos de acesso restrito requer uma licença válida de autorização devendo o utilizador aceder ao(s) documento(s) a partir de um endereço de IP da instituição detentora da supramencionada licença.

Ao utilizador é apenas permitido o descarregamento para uso pessoal, pelo que o emprego do(s) título(s) descarregado(s) para outro fim, designadamente comercial, carece de autorização do respetivo autor ou editor da obra.

Na medida em que todas as obras da UC Digitalis se encontram protegidas pelo Código do Direito de Autor e Direitos Conexos e demais legislação aplicável, toda a cópia, parcial ou total, deste documento, nos casos em que é legalmente admitida, deverá conter ou fazer-se acompanhar por este aviso.

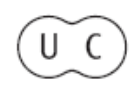




\title{
Utilização da análise multi-níveis para avaliação da vulnerabilidade da população da AML ao calor
}

\author{
Henrique Andrade \\ Centro de Estudos Geográficos. Universidade de Lisboa. \\ henr.andr@gmail.com
}

\section{Helena Nogueira}

Departamento de Geografia da Faculdade de Letras e Centro de Investigação em Antropologia e Saúde. Universidade de Coimbra.

\section{Paulo Canário}

Centro de Estudos Geográficos. Universidade de Lisboa.

\section{Resumo:}

Este estudo analisa o impacte do calor nos níveis de mortalidade da população residente na área metropolitana de Lisboa (AML). Utilizando uma técnica estatística multivariada - análise multi-níveis - procura-se modelar a variação espacial da mortalidade em períodos críticos. Os níveis espaciais considerados na análise foram freguesias e concelhos. Os valores de mortalidade foram padronizados pela idade, utilizando como padrão a população da AML, obtendo-se a razão padronizada de mortalidade (RPM), que constituiu a variável dependente na análise. Como variáveis independentes foram utilizados diferentes indicadores de carácter demográfico, socioeconómico e urbanístico. No primeiro nível (freguesia) as variáveis que se associaram de forma significativa à variação das RPM foram a percentagem de população institucionalizada e o índice de privação sociomaterial; a introdução do nível concelhio na análise incrementou pouco, mas de forma significativa, a explicação da variância das RPM, sendo incluídas na análise o ganho médio e um índice de saúde e assistência social.

Palavras-chave: Calor; mortalidade. Vulnerabilidade. Análise multi-níveis.

\section{Résumé:}

Utilisation de l'analyse multi-niveaux pour évaluer la vulnérabilité à la chaleur de la population de l'AML

Cette étude analyse l'impact de la chaleur sur la mortalité de la population résidant dans l'aire métropolitaine de Lisbonne (AML). En utilisant une technique statistique multivariée - analyse multi-niveaux - cherche à modéliser la variation spatiale de la mortalité au cours des périodes critiques. Les niveaux spatiaux considérés dans l'analyse étaient les communes et les municipalités. Les valeurs de mortalité ont été normalisées selon l'âge, en utilisant comme population de référence de la AML, ce qui donne un ratio standardisé de mortalité (RSM), qui est la variable dépendante dans l'analyse. Comme variables indépendantes ont été utilisées différents indicateurs démographiques, socio-économique et de développement urbain. Au premier niveau (commune) les variables qui ont été associés de manière significative à la variation de RSM sont le pourcentage de la population institutionnalisée et l'indice de privation sociomatériel; l'introduction de l'échelon municipal dans l'analyse augmenté légèrement, mais significativement, l'explication de la variance de SPM, étant inclus dans l'analyse du rendement moyenne et un index des soins de santé et aide social.

Mots-clés: Chaleur. Mortalité. Vulnerabilité. Analyse multi-niveaux.

\section{Abstract:}

Using multi-level analysis to evaluate the vulnerability of the LMA population to heat

This study analyzes the impact of heat on mortality of the resident population in the metropolitan Lisbon area (MLA). Using a multivariate statistical technique - multilevel analysis - we try to model the spatial variation of mortality in critical periods. The spatial levels considered in the analysis were parishes and municipalitys. The mortality values were standardized by age, using as standard population of AML, yielding a standardized mortality ratio (SMR), which is the dependent variable in the analysis. The independent variables were used different indicators of population status, socio-economic and urban development. At the first level (parish) variables that were associated significantly to the 
variation of RPM were the percentage of the institutionalized population and deprivation index sociomaterial; the introduction of the municipal level in the analysis increased slightly, but significantly, the explanation of variance RPM, being included in the analysis the gain medium and an index of health and social care

Keywords: Heat. Mortality. Vulnerability. Multilevel analysis.

\section{Introdução}

Os impactes das vagas de calor sobre a morbilidade e mortalidade das populações têm sido objeto de numerosos estudos (Dessai, 2002; 2003; Díaz et al., 2006; Hutter et al., 2007; Kovats e HAJAT, 2008; TAN et al., 2007; KNowlton et al., 2009), esperando-se que as alterações climáticas aumentem esses impactes (MeEHL e TebaldI, 2004; PATZ et al., 2005; CASIMIRO et al., 2006). A vaga de calor de Agosto de 2003 foi o período mais quente nesta área, desde o início do século XVI (LUTERBACHER et al. 2004), e terá provocado a morte a mais de 70000 pessoas na Europa (RoBine et al. 2008). Em Portugal, o número de óbitos associados a esta vaga de calor terá sido próximo de 2000 (FAlCão et al., 2005). Complementarmente às temperaturas elevadas, considera-se que o ozono $\left(\mathrm{O}_{3}\right)$ é um fator importante nos impactes sobre a saúde em situações de calor (FISCHER et al., 2004; LACOUR et al., 2006; Pelegrini et al., 2007).

0 estudo da relação entre temperatura e mortalidade permite concluir que, em diferentes regiões, os valores mínimos de mortalidade ocorrem em determinados intervalos de temperatura - correspondentes a uma temperatura óptima - acima e abaixo dos quais aumenta o número de óbitos. Os limiares e a amplitude desse intervalo variam regionalmente, refletindo a capacidade de adaptação térmica humana (ALBERDI et al., 1998; CURRIERo et al., 2002; MEdina-Rámon et al., 2006). Há, pois, uma grande variação nesses limiares; por exemplo, em Espanha, em situações de calor, foram identificados limiares para aumento de mortalidade de $26.2^{\circ} \mathrm{C}$, na Galiza e de $41.2^{\circ} \mathrm{C}$, na Andaluzia - uma variação de quase $15^{\circ} \mathrm{C}$ no valor de temperatura associado ao aumento da mortalidade (DIAz et al., 2006).

Os efeitos do calor sobre a saúde têm sido estudados sobretudo a nível nacional ou regional. Existem, contudo, variações espaciais de escala mais fina. Essas dissimetrias espaciais poderão dever-se, por um lado, aos contrastes climáticos locais (por exemplo, os decorrentes da urbanização) e, por outro lado, à desigual vulnerabilidade das populações.

O impacte do calor sobre a saúde varia a nível individual, dependendo, por exemplo, da idade e de outras características demográficas e socioeconómicas; os indivíduos mais sensíveis são sobretudo idosos e aque- les que apresentam patologia prévia específica (Kovats e HAJAT, 2008), associando-se o excesso de mortalidade principalmente a doenças dos aparelhos respiratório e circulatório (PATZ et al. 2005; HAINES et al. 2006; KovATS e HAJAT, 2008). Fatores como o sexo, a capacidade respiratória e cardiovascular, o tamanho e a composição corporal (percentagem de gordura corporal) têm também sido implicados na relação temperatura - mortalidade (HAVENITH, 2005). Para a sensibilidade individual contribuem ainda fatores culturais, sociais e económicos, como o isolamento/suporte social e o estatuto socioeconómico; a sensibilidade aumenta para indivíduos e grupos da base da hierarquia social, o que se compreende atendendo a fatores como o tipo de ocupação (predominantemente manual, envolvendo esforço físico), as piores condições de trabalho, o mais difícil acesso à informação e o menor acesso aos serviços de saúde, entre outros (Kovatz e HAJAT, 2008).

Para além da sensibilidade individual, os impactes dos extremos térmicos na mortalidade relacionam-se com o nível de exposição de cada indivíduo ao evento térmico. Por sua vez, a exposição decorre quer da mobilidade individual, quer das características dos locais de trabalho e de residência, como a qualidade da habitação e a estrutura verde. A exposição está, pois, relacionada com a sensibilidade: o estatuto socioeconómico condiciona não apenas o tipo de ocupação e o acesso à informação, mas também a qualidade da habitação, as características do local de residência e a mobilidade; a idade e o sexo relacionam-se também com a mobilidade.

A avaliação da vulnerabilidade aos extremos térmicos deve então considerar aspetos relativos à sensibilidade, à exposição e ainda as inter-relações existentes entre estes: por exemplo, a vulnerabilidade de um indivíduo idoso pode resultar de alterações fisiológicas decorrentes do envelhecimento biológico, do isolamento social, da existência de patologia respiratória ou cardíaca, do baixo rendimento, que condiciona as condições da habitação e a capacidade de pagar cuidados de saúde, da reduzida mobilidade. Alguns destes fatores são potenciados pelo sexo; por exemplo, as mulheres são mais sensíveis ao calor, devido a fatores hormonais ( $\mathrm{H}_{\text {AVENITH, }}$ 2005), sendo também mais provável que, dada a sua maior longevidade, se encontrem socialmente isoladas. 
Todavia, muitas das variáveis subjacentes à vulnerabilidade (sensibilidade e exposição) são redundantes, podendo e devendo ser sintetizadas através de indicadores compósitos de vulnerabilidade. Estas medidas têm conhecido um amplo desenvolvimento e têm sido aplicadas tanto na análise dos impactes dos extremos térmicos na saúde, como em estudos mais genéricos de variações espaciais da saúde. Considerando a sensibilidade ao calor como um componente da vulnerabilidade, McGregor et al. (2007) desenvolveram um índice compósito de sensibilidade, que aplicaram a 4765 unidades espaciais de Londres. As variáveis consideradas nesse índice foram: percentagens de população idosa (entre 65 e 84 anos) e muito idosa (mais de 85 anos), percentagem de população com doença incapacitante de longa duração, percentagem de população institucionalizada (em estabelecimentos prestadores de cuidados médicos e sociais), percentagem de população residente em alojamento partilhado (apartamento); nível de privação da população (índice de privação múltipla que inclui uma variável relacionada com a educação), percentagem de famílias com residência a partir do quinto andar (inclusive), percentagem de famílias constituídas por reformados vivendo sós, densidade populacional. As variáveis foram classificadas em decis, aos quais foi atribuída uma pontuação entre 1 a 10 . 0 índice de sensibilidade resultou da acumulação das pontuações dos decis para as nove variáveis, podendo teoricamente variar entre um valor mínimo de 9 a um valor máximo 90 .

No nosso país, não foi ainda criada uma medida que permita avaliar a sensibilidade da população aos fatores climáticos; no entanto, refira-se que algumas medidas compósitas de vulnerabilidade têm sido desenvolvidas e aplicadas a estudos de variações em saúde. Uma destas medidas foi desenvolvida para avaliar a privação múltipla nas freguesias da Área Metropolitana de Lisboa ( $A M L)$, relacionando-a com a mortalidade prematura, concluindo-se pela forte associação entre privação socioeconómica e mortalidade prematura (NoGUEIRA, 2007).
Neste trabalho pretende-se aplicar a análise multi-níveis (método estatístico correntemente utilizado em epidemiologia - Leyland e Groenewegen, 2003; Cho, 2003) no desenvolvimento de um modelo espacial de vulnerabilidade ao calor na AML, à escala local. 0 trabalho está ainda em desenvolvimento, apresentando-se aqui a metodologia seguida e os primeiros resultados.

\section{Material e métodos}

Neste estudo foram utilizados dados de mortalidade diária por todas as causas, por freguesia, por sexo e grupo etário, entre 1998 e 2008, fornecidos pelo Instituto Nacional de Estatística (INE). Os dados de temperatura (obtidos através do National Climatic Data Center) são da estação meteorológica de Lisboa/ Gago Coutinho, correntemente considerada como representativa da região de Lisboa (ANDRADE e AlCOFORADO, 2006). Os indicadores de vulnerabilidade foram obtidos do INE (Censo de 2001) e de algumas outras fontes. Os indicadores considerados inicialmente são enumerados no Quadro I.

O índice de privação sociomaterial (NogueIRA, 2007) foi calculado a partir da taxa de desemprego masculina, da percentagem de emprego não qualificado e da percentagem de indivíduos a viver em habitações com condições precárias.

Para a avaliação da vulnerabilidade ao calor foram selecionados os dias com temperatura máxima superior ou igual a $32^{\circ} \mathrm{C}$ (critério baseado em NogueIRA e PAIXÃo, 2008). De acordo com este critério, foram selecionados 192 dias entre Maio e Setembro, sendo a temperatura máxima absoluta atingida nesses dias $41,7^{\circ} \mathrm{C}$, no dia 1 de Agosto de 2003.

A análise foi desenvolvida ao nível da freguesia (211 no total); contudo, para evitar resultados estatisticamente inconsistentes em freguesias de muito pequena dimensão, procedeu-se à agregação daquelas que têm uma população inferior a 1500 habitantes. Esse processo afetou sobretudo as freguesias da área histó-

Quadro I

Indicadores de vulnerabilidade

\begin{tabular}{|c|c|}
\hline Grupos & Indicadores \\
\hline Indicadores demográficos & $\%$ de viúvos ${ }^{1}$, taxa de feminilidade ${ }^{1}$, densidade populacional ${ }^{1}$ \\
\hline Indicadores educacionais & taxa de analfabetismo ${ }^{1}, \%$ de população com ensino superior completo ${ }^{1}$ \\
\hline Indicadores socioeconómicos & $\begin{array}{l}\text { ganho médio }{ }^{1}, \% \text { de institucionalizados }{ }^{1}, \% \text { de dependentes de apoio social }{ }^{1}, \% \text { profissões socialmente mais valorizadas }{ }^{1} \text {, índice } \\
\text { de privação sociomaterial }{ }^{*}\end{array}$ \\
\hline Indicadores urbanísticos & densidade edifícios $^{1}$, idade média dos edifícios ${ }^{1}, \%$ área construída ${ }^{2}$, densidade populacional líquida ${ }^{12}$ \\
\hline Indicadores de saúde & distância aos hospitais ${ }^{3}$, índice de saúde e assistência social (ISAS) ${ }^{4}$ \\
\hline
\end{tabular}

Fontes: 1. INE * calculado de acordo com Nogueira (2007); 2. Corine; 3. SAntana et al., 2004; 4. FonseCa (2003) 
rica de Lisboa e algumas das áreas rurais periféricas, tendo este processo reduzido a 180 o número de unidades de análise.

De acordo com análises anteriores, a estrutura etária da população e os fatores relacionados com esta - nomeadamente a percentagem de viúvos, o que reflete a importância do isolamento físico e social no impacte do calor - são as principais condicionantes da vulnerabilidade ao calor. Com o objetivo de comparar as diferentes unidades de análise, excluindo o efeito da estrutura etária, foram calculadas razões padronizadas de mortalidade (RPM) (NogueIRA, 2007), A taxa de mortalidade padronizada é ajustada para diferenças na composição entre a população ou território estudado e uma população padrão (Nogueira, 2008). Fez-se aqui a padronização pelo método indirecto, recorrendo a taxas de mortalidade específicas por idade da população padrão (a da $A M L$ ), estimando, a partir delas, os óbitos esperados numa dada região (NogueIRa e Remoaldo, 2010). Valores superiores a 100 revelam uma mortalidade superior à verificada na população padrão - 100 enquanto valores inferiores assinalam uma mortalidade inferior à dessa população. A RPM foi calculada para população total e para a população com idade superior a 65 anos (Figura 1). O valor da RPM variou entre 33.8, na freguesia de Alfornelos (Amadora) e 172.2, na freguesia do Socorro (Lisboa).
Na análise da relação entre a RPM e os indicadores de vulnerabilidade foi utilizada a análise multi-níveis, cujo esquema conceptual é apresentado na Figura 2. Neste modelo, também designado como modelo linear hierárquico, são considerados diferentes níveis de análise, em que cada um explica uma parte da variância não explicada pelo nível anterior (Leyland e Groenewegen, 2003; Cho, 2003). O modelo permite selecionar, em cada nível, as variáveis que se relacionam significativamente com a variável dependente (sendo a significância avaliada através do $p$-value). 0 teste de deviance é utilizado para testar o modelo geral, podendo ser considerada como medida da falta de ajustamento entre o modelo e os dados (С $\mathrm{Ho}, 2003)$, sendo por isso o ajustamento tanto maior quanto menor o valor do teste. Complementarmente, foi feita a análise dos resíduos em cada nível.

Neste estudo foram considerados dois níveis: o da freguesia e o do concelho; a análise foi feita utilizando o software MIWin.

\section{Resultados e discussão}

Os resultados da análise são apresentados no Quadro II.

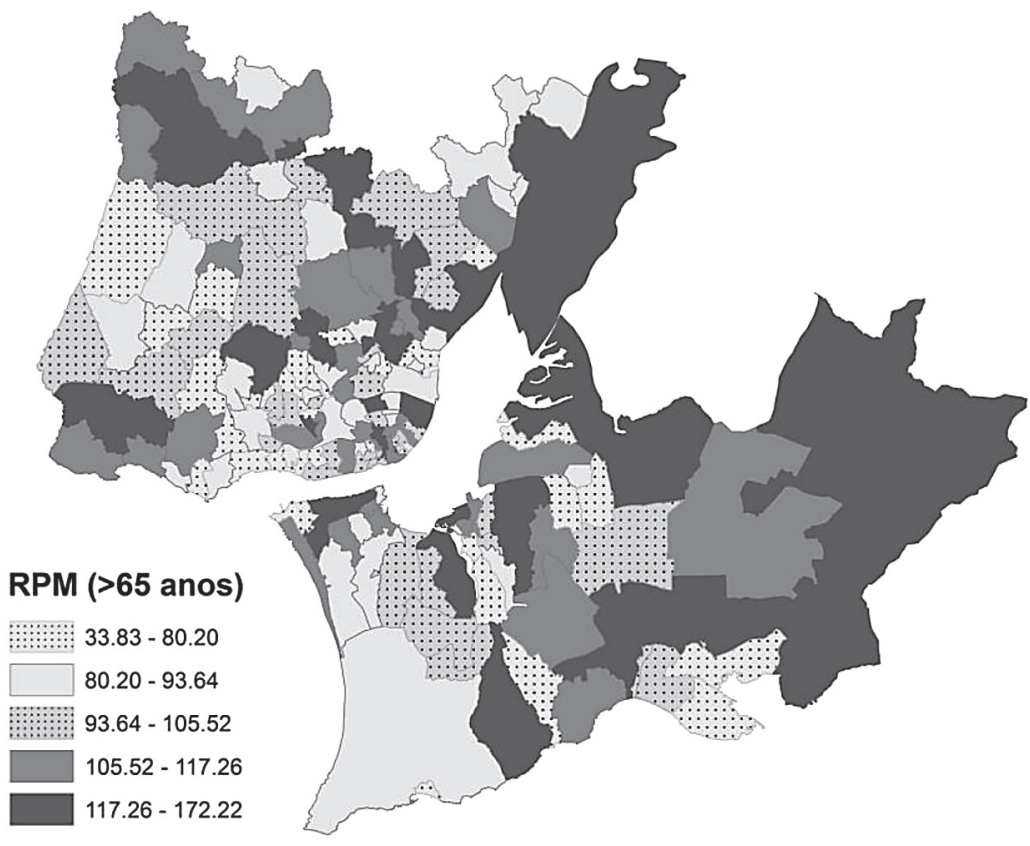

Figura 1

Razões padronizadas de mortalidade na AML 


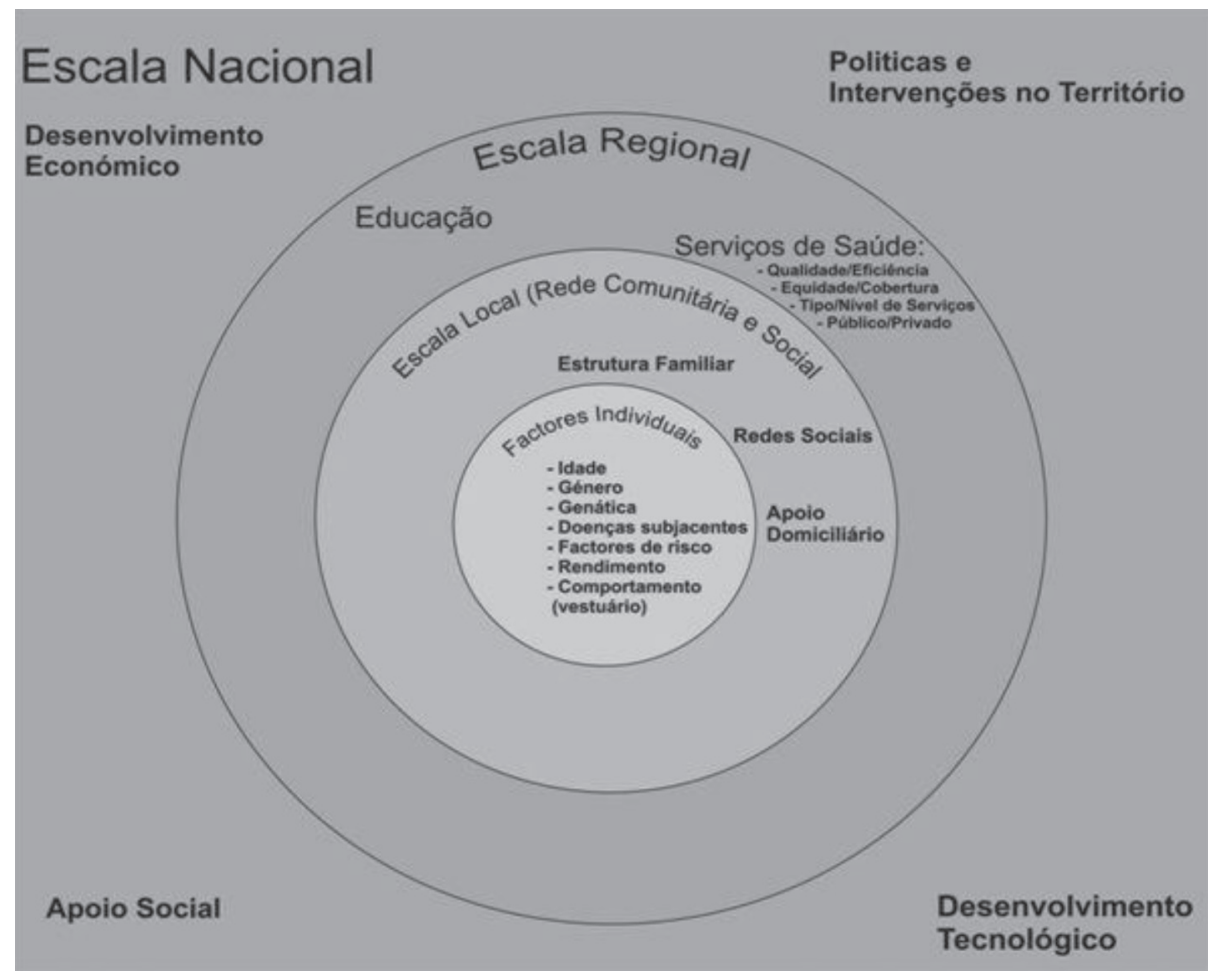

Figura 2

Modelo conceptual da análise multiníveis (CANÁRIO, 2010)

Considerando o primeiro nível, as duas variáveis com relação significativa com as RPM são a percentagem de população institucionalizada, a qual reflete, em grande parte, a estrutura etária da população e também, em menor grau, o seu estado de saúde e o índice de privação sociomaterial, indicador compósito das condições socioeconómicas. Em qualquer caso, a relação com o índice de privação sociomaterial revela o peso das variáveis socioeconómicas na variação da RPM.

Ao acrescentar o segundo nível de análise verifica-se uma pequena mas significativa diminuição da deviance e um pequeno aumento $(1.64 \%)$ do ajustamento entre os valores estimados e observados. As variáveis significativas ao nível do concelho foram o ganho médio (indicador do nível económico médio da população) e o ISAS, indicador compósito feito a partir de diferentes variáveis.

\section{Conclusão}

A variância das RPM é explicada principalmente pelas características demográficas e socioeconómicas da população residente nas freguesias da $A M L$, sendo

\section{Quadro II}

Resultados da análise multi-níveis

\begin{tabular}{|l|l|l|}
\hline Nível 1 & Deviance & 1616.898 \\
\hline & Ajustamento dos valores estimados aos observados (\%) & 81.3 \\
\hline & Variáveis significativas & Percentagem da população institucionalizada em instituições de apoio social e de saúde \\
\hline & & Índice de privação sociomaterial \\
\hline Nível 2 & Deviance & 1592.445 \\
\hline & Ajustamento dos valores estimados aos observados (\%) & 82.96 \\
\hline & Variáveis significativas & Ganho médio \\
\hline & & Índice de saúde e assistência social (ISAS) \\
\hline
\end{tabular}


o ajustamento entre valores estimados e observados bastante elevado $(81,3 \%)$; conclui-se que as freguesias mais favorecidas em termos socioeconómicos e com menor percentagem de população institucionalizada apresentaram menores RPM nos períodos críticos considerados (dias com temperaturas máximas iguais ou superiores a $32^{\circ} \mathrm{C}$ ). A nível concelhio, o ganho médio e o ISAS contribuem com cerca de 1,66\% para a explicação da variância das RPM, valor que não sendo muito elevado é, ainda assim, estatisticamente significativo.

\section{Bibliografia}

Alberdi, J.; Diaz, J.; Montero, J. e Miron, I. (1998) -"Daily mortality in Madrid community 1986-1992: relationship with meteorological variables”. Eur J Epidemiol 14(6), pp. 571-578.

Andrade , H. e Alcoforado, M. J. (2008) -"Microclimatic variation of thermal comfort in a city-district of Lisbon (Telheiras). Night-time period”. Theor Appl Climatol, 92, pp. 225237.

CanÁrIO, P. (2010) - "Methodology to assess thermal extremes mortality risk in urban areas". Finisterra - Revista Portuguesa de Geografia, XLIV, 89, pp. 167-173.

Casimiro, E.; Calheiros, C.; Santos, F. D. e Kovats, S. (2006) "National assessment of human health effects of climate change in Portugal: approach and key findings". Environ Health Perspect, 114, pp. 1950-1956.

CHO, H. (2003) - "Using multilevel analysis in patient and organizational outcomes research". Nurs Res, 52, pp. 61-65.

Contı, S.; Meli, P.; Minelli, G.; Solimini, R.; Toccaceli, V.; Vichi, M.; Beltrano, C. e Perini, L. (2005) - "Epidemiologic study of mortality during the summer 2003 heat wave in Italy". Environ Res, 98, pp. 390-399.

Curriero, F.; Heiner, K.; Samet, J.; Zeger, S.; Strug, L. e Patz, J. (2002) - "Temperature and mortality in 11 cities of the eastern United States”. Am J Epidemiol, 155, pp. 80-87.

Dessal, S. (2002) -"Heat stress and mortality in Lisbon Part I Model construction and validation". Int J Biometeorol, 47, pp. 6-12.

DESSAI, S. (2003) -"Heat stress and mortality in Lisbon Part II. An assessment of the potential impacts of climate change". Int J Biometeorol, 48, pp. 37-44.

Diaz, J.; García-Herrera, R.; Trigo, R.; Linares, C.; Valente, M.; Miguel, J. e Hernández, E. (2006) - "The impact of the summer 2003 heat wave in Iberia: how should we measure it?" Int J Biometeorol, 50, pp. 159-166.

Ebi, K.; Mills, D.; Smith, J. e Grambsch, A. (2006) - "Climate Change and Human Health Impacts in the United States - An
Update on the Results of the U.S. National Assessment”. Environmental Health Perspectives, 114, pp. 1318-1324.

Falcão, P.; Contreiras, J.; Paixão, M.; Brandão, E. e Batista, I. (2005) - "Mortality in Portugal associated with the heat wave of august 2003: early estimation of effect, using a rapid method". Eurosurveillance, 10, pp. 150-153.

Fischer, P.; BRUneKReEF, B. e Lebret, E. (2004) - "Air pollution related deaths during the 2003 heat wave in the Netherlands". Atmospheric Environment, 38, pp. 1083-1085

FonseCA, P. (2003) - Índices de desenvolvimento concelhio. Lisboa, INE.

Haines, H.; Kovats, R. e Corvalan, C. (2006) - "Climate change and human health - impacts, vulnerability, and mitigation". Lancet, 367, pp. 2101-2109.

HAVENITH, G. (2005) - "Temperature Regulation, Heat Balance and Climatic Stress”. In: KIRCH, B. Menne e BertollinI, R. (Eds.)

- Extreme Weather Events and Public Health. Berlin Springer, pp. 59-67.

Hutter, H.; Moshammer, H.; Wallner, P.; Leitner, B. e Kundi, M. (2007) - " Heatwaves in Vienna: effects on mortality". Wien Klin. Wochenschr, 119, pp. 223-227.

Knowlton, K.; Rotkin-Ellman, M.; King, G.; Margolis, H. G.; SMith, D.; Solomon, G.; Trent, R. e English, P. (2009) - "The 2006 California Heat Wave: Impacts on Hospitalizations and Emergency Department Visits". Environ Health Persp, 117(1), pp. 61-67.

Kovats, R. S. e HaJat S. (2008) - "Heat Stress and Public Health: A Critical Review”. Annual Review of Public Health, 29 pp. 41-55.

Lacour, S.; De Monte, M.; Diot, P.; Brocca, J.; Veron, N.; Colin, P. e Leblond, V. (2006) -" Relationship between ozone and temperature during the 2003 heat wave in France: consequences for health data analysis". BMC Public Health, 6, pp. 261.

LeYland, A. e Groenewegen, P. (2003) - "Multilevel modelling and public health policy". Scand J Public Health, 31, pp. 267-274

Lopez, A.; Mathers, C.; Ezzati, M.; Murray, C. e Jamison, D. (2006)

- Global burden of disease and risk factors. New York, Oxford University Press.

Luterbacher, J.; Dietrich, D.; Xoplaki, E.; Grosjean, M. e Wanner, H. (2004) - "European Seasonal and Annual Temperature Variability, Trends, and Extremes Since 1500”. Science, 303, pp. 1499-1503.

McGregor, R.; Pelling, M.; Wolf, T. e Gosling, S. (2007) -The social impacts of heat waves. Environment Agency, Rio House, Waterside Drive.

Medina-Rámon, M.; Zanobetti, A.; Cavanagh, D. P. e Schwartz, J. (2006) - "Extreme Temperatures and Mortality: Assessing Effect Modification by Personal Characteristics and Specific Cause of Death in a Multi-City Case-Only Analysis”. Environmental Health Perspectives, 114-9, pp. 1331-1336. 
MeEHL, G. e TebaldI, C. (2004) - "More Intense, More Frequent, and Longer Lasting Heat Waves in the 21st Century". Science, 305, pp. 994-997

Metzger M. J. e Schröter, D. (2006) -"Towards a spatially explicit and quantitative vulnerability assessment of environmental change in Europe". Reg Environ Change, 6, pp. 201-216.

NogueiRa, H. (2007) - "Privação sociomaterial e saúde na Área Metropolitana de Lisboa". In: Santana, P. (coord.) - A Cidade e a Saúde. Coimbra. Edições Almedina, Coimbra, p. $155-162$.

Nogueira, H. e Remoaldo, P. (2010) - "Olhares Geográficos sobre a saúde". Lisboa, Edições Colibri.

Nogueira, P. e PaIX̃̃o, E. (2008) - Models for mortality associated with heatwaves: update of the Portuguese heat health warning system". Int. J. Climatol., 28, pp. 545-562.
Patz, J.; Campbell-Lendrum, C.; Holloway, T. e Foley, J. A. (2005) - "Impact of regional climate change on human health". Nature, 438, pp. 310-317.

Pellegrini, E.; Lorenzini, G. e Nal, C. (2007) - "The 2003 European Heat Wave: Which Role for Ozone? Some Data from Tuscany, Central Italy". Water Air Soil Pollut, 181, pp. 401-408.

Robine, J.; Cheung, S.; Sophie, L.; Herman, V.; Clare, G.; Michel, J. e Herrmann, F. (2008) -"Death toll exceeded 70,000 in Europe during the summer of 2003. C. R. ". Biologies, 331, pp. 171-178.

Santana, P.; Pereira, N. e Nogueira, H. (2004) - Carta de Equipamentos de Saúde - Centros de Saúde. Região de Lisboa e Vale do Tejo. Lisboa, Direcção-Geral da Saúde

Tan, J.; Zheng, Y.; Song, G.; Kalkstein, L. S.; Kalkstein, A. e Tang, X. (2007) - "Heat wave impacts on mortality in Shanghai, 1998 and 2003". Int J Biometeorol, 51, pp. 193-200. 